\title{
Untersuchungen zur Gleichgewichtsfeuchte und Quellung von Massivholzplatten
}

Online veröffentlicht: 23 März 2004

(C) Springer-Verlag 2004

Zusammenfassung Aus den Untersuchungen an mehrschichtigen Massivholzplatten aus Fichtenholz geht hervor, dass die Gleichgewichtsfeuchtigkeit im unteren hygroskopischen Bereich bis etwa 65\% rel. Luftfeuchtigkeit bedeutend höher und im obersten Bereich bedeutend tiefer ist als diejenige beim Fichtenholz. Die Messungen haben gezeigt, dass die Längen- sowie die Breitenquellung gegenüber der Dickenquellung vernachlässigbar klein ist, sodass die Volumenquellung der Dickenquellung gleichgestellt werden kann. Das Quellen im Bereich zwischen $0 \%$ und etwa $20 \%$ Wassergehalt ist nahezu linear. Diese Beziehung korreliert mit der Sorptionsisotherme. Die Quellung steigt linear mit der Anzahl Leimschichten pro Plattendicke, wobei die Steigung der Geraden vom Bereich der Chemi- über die Physisorption bis zum Bereich der Kapillarkondensation stetig ansteigt.

\section{Equilibrium moisture content and swelling of the solid wood panels}

Abstract The investigations on multilayer solid spruce wood panels showed that the equilibrium moisture content in the hygroscopic range until $65 \%$ relative humidity is significantly higher and in the upper range lower than in spruce wood. From these measurements it is concluded that the length- and the width swelling are negligibly small in relation to the thickness swelling, so that the thickness swelling can be set equal to the volume swelling. Swelling within the range between $0 \%$ and about 20\% moisture content is almost linear and the relationship between swelling and relative humidity corresponds approximately to the shape of the sorption isotherm. The swelling increases linear with the number of adhesive layers per panel thickness, whereby slope of the straight lines rises constantly from the range of the chemo- over the physisorption up to the range of the capillary condensation.

R. Popper · P. Niemz $(\bowtie) \cdot$ G. Eberle

D-BAUG Gruppe Holzphysik, ETH Zuerich,

ETH-Zentrum, 8092 Zürich, Schweiz

E-Mail: niemz@ibwk.baug.ethz.ch

\section{Einleitung}

Mehrschichtige Massivholzplatten aus Nadelholz werden zunehmend im Bauwesen eingesetzt. Kennwerte für die mechanisch-physikalischen Eigenschaften aber auch das Quell- und Schwindverhalten liegen bisher nur in geringem Umfang, meist aus Firmenschriften vor (siehe Fa. P.Schuler sowie Radoviz, Cheret, Heim (1997)). Umfangreiche Arbeiten zu Massivholzplatten wurden im IHD Dresden durchgeführt (siehe z.B. Tobisch 1999a, b). Die Werte liegen bei Massivholzplatten für die Längenquellung etwa bei $0,015 \% / \%$ Feuchteänderung, für die Dickenquellung bei $0,5 \% / \%$.

Vergleichsweise werden für harnstoffharzverleimte Spanplatten $0,035 \% 1 \%$ (Längenänderung) bzw $0,7 \% / \%$ (Dickenänderung) und für phenolharzverleimte 0,025/\% (Längenänderung) und 0,45\%/\% (Dickenänderung) angegeben. Bei MDF ermittelten Niemz und Poblete (1996) etwas niedrigere Werte $(0,019 \% / \%)$ als bei Spanplatten $(0,032 \% / \%)$. Dies deckt sich mit Messungen von Suchland und Xu (1991). Bei Sperrholz liegt der Wert für die Längenquellung etwa bei $0,02 \% / \%$, für die Dickenquellung bei $0,3 \% / \%$. Vergleichsweise gibt Sell (1989) für Fichtenholz radial $0,15 \ldots 0,19$ und tangential $0,27 . .0,36$ an. Die Längenquellung beträgt bei Fichte etwa $0,01 \% / \%$.

\section{Material und Methodik}

\subsection{Versuchsmaterial}

Als Versuchsmaterial (Tab. 1) dienten vier industriell gefertigte Massivholzplatten (mit PUR verklebt) mit verschiedenem Plattenaufbau und Fichtenholz (Abb. 1). Zusätzlich wurde eine Betonschaltafel auf Massivholzplattenbasis (verklebt mit Melaminharz, Oberflächenbeschichtung mit Melamin, Mittellage geschlitzt) getestet. Die Prüfung erfolgte in Anlehnung an EN 318 an Proben mit $200 \mathrm{~mm}$ Länge und $20 \mathrm{~mm}$ Breite. Es wurden jeweils 10 Proben mit Orientierung der Decklagen parallel und senkrecht zur Probenlängsachse geprüft. 


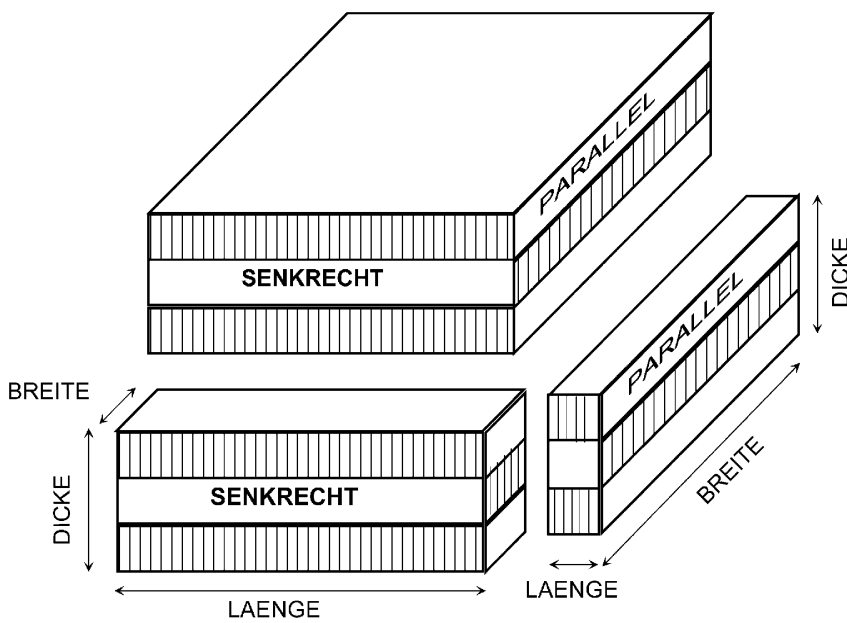

Abb. 1 Aufbau der Platten

Fig. 1 Structure of the panels

\subsection{Versuchsdurchführung}

Zur Einstellung der Luftfeuchtigkeit dienten Behälter mit gesättigten Salzlösungen gemäß Tabelle 2. Die Temperatur betrug $20^{\circ} \mathrm{C}$. Die Proben wurden bis zum Erreichen der Gleichgewichtsfeuchte im jeweiligen Klima gelagert. Die Holzfeuchte wurde mittels Darrmethode bestimmt. Die Messung der Längenänderung erfolgte mit einem inkrementalen Wegaufnehmer. Als Bezugslänge der Quellung diente der darrtrockene Zustand. Die in 2.2.12.2.3 aufgeführten Kenngrößen wurden geprüft.

\subsubsection{Feuchtigkeitsgehalt}

Der prozentuale Feuchtigkeitsgehalt der Probe $u$ ist das Verhältnis zwischen der Masse des in der Probe enthaltenen Wassers und der Masse der wasserfreien (darrtrockenen) Probe. Für den Feuchtigkeitsgehalt gilt nach DIN 52183 (1977):

$u=\frac{m_{u}-m_{0}}{m_{0}} \times 100$ in $\%$,

wobei

$m_{\mathrm{u}} \quad$ Masse der feuchten Probe in $g$,

$m_{0} \quad$ Masse der wasserfreien (darrtrockenen) Probe in $g$.

\subsubsection{Feuchteverformung/Quellung}

\subsubsection{Das maximale lineare Quellmaß}

Das maximale Quellmaß $\alpha_{\max }$ ergibt sich bei Quellung vom darrtrocknenen auf den feuchten Zustand des Materials. Je nach dem für welche Richtung (Dicke $D$, Breite $B$ und Länge $L$ ) und Feuchtigkeitsgehalt $u$ der Versuchsprobe die Ausmaße $a$ ermittelt werden, unterscheidet man zwischen dem Dicken- $\alpha_{D u}$, dem Breiten- $\alpha_{B u}$ und dem Längen-Quellmaß $\alpha_{L u}$ :

Für die Dickenquellung gilt z. B.

$\alpha_{\max , D u}=100 \times \frac{\left(a_{D u}-a_{D 0}\right)}{a_{D 0}}$ in $\%$

Tabelle 1 Versuchsübersicht

Table 1 Test outline

\begin{tabular}{|c|c|c|c|c|c|c|}
\hline Bezeichnung & Plattentyp & $\begin{array}{l}\text { Anzahl } \\
\text { Schichten }\end{array}$ & Dicke mm & Lamellen-verhältnis & $\begin{array}{l}\text { Anordnung der } \\
\text { Decklagen }\end{array}$ & Bemerkung \\
\hline MHP-3-60P & Massivholzplatten & 3 & 60 & $20 / 20 / 20$ & Parallel & \\
\hline MHP-3-60S & Massivholzplatten & 3 & 60 & $20 / 20 / 20$ & Senkrecht & \\
\hline MHP-3-27S & Massivholzplatten & 3 & 27 & $6 / 15 / 6$ & Senkrecht & $\begin{array}{l}\text { Beidseitig beschich- } \\
\text { tet. Mittellamelle } \\
\text { geschlitzt }\end{array}$ \\
\hline MHP-3-30P & Massivholzplatten & 3 & 30 & $10 / 10 / 10$ & Parallel & \\
\hline MHP-3-30S & Massivholzplatten & 3 & 30 & $10 / 10 / 10$ & Senkrecht & \\
\hline MHP-3-60AP & Massivholzplatten & 3 & 60 & $10 / 40 / 10$ & Parallel & \\
\hline MHP-3-60AS & Massivholzplatten & 3 & 60 & $10 / 40 / 10$ & Senkrecht & \\
\hline MHP-5-60P & Massivholzplatten & 5 & 60 & $12 / 12 / 12 / 12 / 12$ & Parallel & \\
\hline MHP-5-60S & Massivholzplatten & 5 & 60 & $12 / 12 / 12 / 12 / 12$ & Senkrecht & \\
\hline
\end{tabular}

Tabelle 2 Die verwendeten Klimata und ihre Erzeugung Table 2 The climates applied and their generation

\begin{tabular}{lcc}
\hline Klimaerzeugung & Rel. Luftfeuchtigkeit $(\%)$ & Temperatur $\left({ }^{\circ} \mathrm{C}\right)$ \\
\hline Darrtrocknung & 0 & 105 \\
$\mathrm{LiCl}$ & 11 & 20 \\
$\mathrm{~K}_{2} \mathrm{CO}_{3}$ & 44 & 20 \\
$\mathrm{Klimaum}_{\mathrm{NaCl}}$ & 65 & 20 \\
$\mathrm{NH}_{4} \mathrm{H}_{2} \mathrm{PO}_{4}$ & 75 & 20 \\
\hline
\end{tabular}


Analog berechnet sich die Breiten und Längenquellung. Das Volumenquellma $\beta$ ist als Summe aus dem maximalen Dicken-, Breiten- und Längen-Quellmaß definiert (Gl. 3).

$\alpha_{\max , V u}=\alpha_{\max , D u}+\alpha_{\max , B u}+\alpha_{\max , L u}$ in $\%$

wobei

$a_{\text {Du }}$ Dickenabmessung der Platte bei Materialfeuchte $u$, $a_{\mathrm{D} 0}$ Dickenabmessung der darrgetrockenen Platte,

$a_{\mathrm{Bu}}$ Breitenabmessung der Platte bei Materialfeuchte $u$, $a_{\mathrm{B} 0}$ Breitenabmessung der darrgetrockenen Platte,

$a_{\text {Lu }}$ Längenabmessung der Platte bei Materialfeuchte $u$, $a_{\mathrm{L} 0}$ Längenabmessung der darrgetrockenen Platte.

\subsubsection{Differentielle Quellung}

In der Praxis wird häufig die differentielle Quellung verwendet. Diese ist gemäß DIN 52184 als das prozentuale Quellmaß der Probe je 1\% Holzfeuchtigkeitsänderung wie folgt definiert:

$q=\frac{a_{F}-a_{T}}{a_{0} \cdot\left(u_{F}-u_{T}\right)} \times 100$ in $\% / \%$

wobei

$q$ die differentielle Quellung in $\% / \%$,

$a_{\mathrm{F}} \quad \mathrm{Ma} ß$ der Probe im feuchten Klima nach Erreichen des Gleichgewichtszustandes in $\mathrm{mm}$,

$a_{\mathrm{T}} \quad \mathrm{Ma} \beta$ der Probe im trockenen Klima nach Erreichen des Gleichgewichtszustandes in mm,

$a_{0} \quad \mathrm{Ma} ß$ der Probe im darrtrockenen Zustand in $\mathrm{mm}$,

$u_{\mathrm{F}} \quad$ Feuchtigkeitsgehalt des Probe im feuchten Klima nach Erreichen des Gleichgewichtszustandes in \%,

$u_{\mathrm{T}} \quad$ Feuchtigkeitsgehalt des Probe im trockenen Klima nach Erreichen des Gleichgewichtszustandes in \%.

Als Feuchtklima wurde die relative Luftfeuchtigkeit von $93 \%$ bei einer Temperatur von $20^{\circ} \mathrm{C}$ und als Trockenenklima die relative Luftfeuchtigkeit von $44 \%$ bei einer Temperatur von $20^{\circ} \mathrm{C}$ gewählt.

\subsubsection{Wasserdampfsorption}

Für die Charakterisierung der Wechselwirkung zwischen dem Versuchsmaterial und dem Wasserdampf, wurde das Hailwood-Horrobin Sorptionsmodell (1946) verwendet. Das Hailwood-Horrobin Sorptionsmodell (weiter als HHSorptionsmodell) basiert auf der Annahme, dass das Wasser bei der Wasserdampfsorption durch Lignocellulosen als einfache Lösung und als Hydrat der Lignocellulosen existiert. Es wird weiter angenommen, dass die sorbierte Schicht, die aus nicht hydratisierten und hydratisierten Lignocellulosen sowie aus freiem flüssigem Wasser besteht, eine ideale feste Lösung bildet. Durch das HH-Modell lassen sich noch weitere Größen schätzen, so die Unzugänglichkeit der sorptiv aktiven Stellen des Sorbens zum Sorbat $(Z)$, ferner das hypothetische Mole- kulargewicht des Sorbens $\left(M_{P}\right)$. Für die experimentell schwer zu bestimmende Fasersättigungsfeuchtigkeit $\left(U_{F S}\right)$ lässt sich eine Schätzgröße errechnen. Weiter wird das monomolekular $\left(U_{m}\right)$ und das polymolekular $\left(U_{p}\right)$ gebundene Wasser durch das HH-Sorptionsmodell ermittelt. Die Berechnung der aus dem HH-Sorptionsmodell abgeleiteten Größen erfolgte mit dem Computerprogramm HHM28 (Popper 1982).

\section{Versuchsergebnisse}

\subsection{Sorptionseigenschaften}

Die Abb. 2, 3, 4, 5 und 6 zeigen exemplarisch die gemessenen, sowie die nach HH-Sorptionsmodell berechneten Isothermen ausgewählter Materialien und zum Vergleich dazu von Fichtenholz (Abb. 6). Dargestellt sind in diesen Abbildungen ebenfalls die Bereiche der Sorbens/Sorbat-Interaktion (Chemi-, Physisorption und die Kapillarkondensation). Jeder Punkt auf der Grafik entspricht einem Mittelwert von 10 Versuchsproben (Tabelle 3).

Die aus dem HH-Sorptionsmodell abgeleiteten physikalischen Größen sind in Tabelle 4 zusammengefasst. Es ergab sich eine sehr gute Übereinstimmung der Messwerte mit den durch das Hailwood-Horrobin Modell berechneten Isothermen.

Wie aus der Tabelle 4 ersichtlich ist besteht kein relevanter Unterschied zwischen den Sorptionseigenschaften der verschiedenen Massivholzplatten. Eine tendentielle

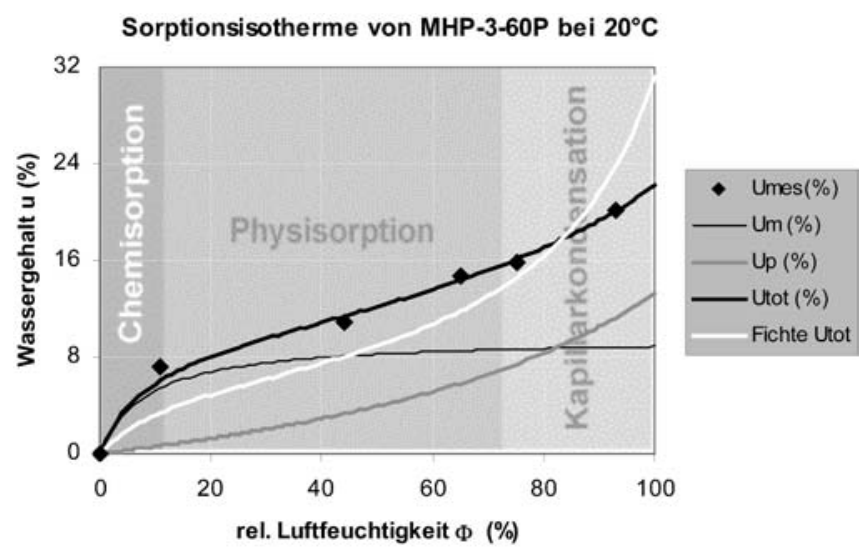

Abb. 2 Sorptionsisotherme von 3-schichtigen (Lamellenverhältnis 20/20/20), $60 \mathrm{~mm}$ dicken Massivholzplatten in paralleler Anordnung bei $20^{\circ} \mathrm{C}$. $U_{m e s}$ - gemessener Wassergehalt der Proben beim rel. Dampfdruck $\phi$ in \%; $U_{m}$-berechneter Wassergehalt in der monomolekularen Schicht beim rel. Dampfdruck $\phi$ in $\% ; U_{p}$ berechneter Wassergehalt in der polymolekularen Schicht beim rel. Dampfdruck $\phi$ in $\%, U_{t o t}$-berechneter Wassergehalt beim rel. Dampfdruck $\phi$ in $\%$

Fig. 2 Sorption isotherm of three layer solid wood panels (layer arrangement 20/20/20), $60 \mathrm{~mm}$ thick parallelly arranged. $U_{m e s}-$ moisture content (MC) measured at relative humidity $\phi(\%) ; U_{m}-$ computed $\mathrm{MC}$ in the monomolecular layer at rel. humidity $\phi(\%)$; $U_{p}$-computed MC in the polymolecular layer at rel. humidity $\phi$ $(\%), U_{t o t}-$ computed $\mathrm{MC}$ at rel. humidity $\phi(\%)$ 


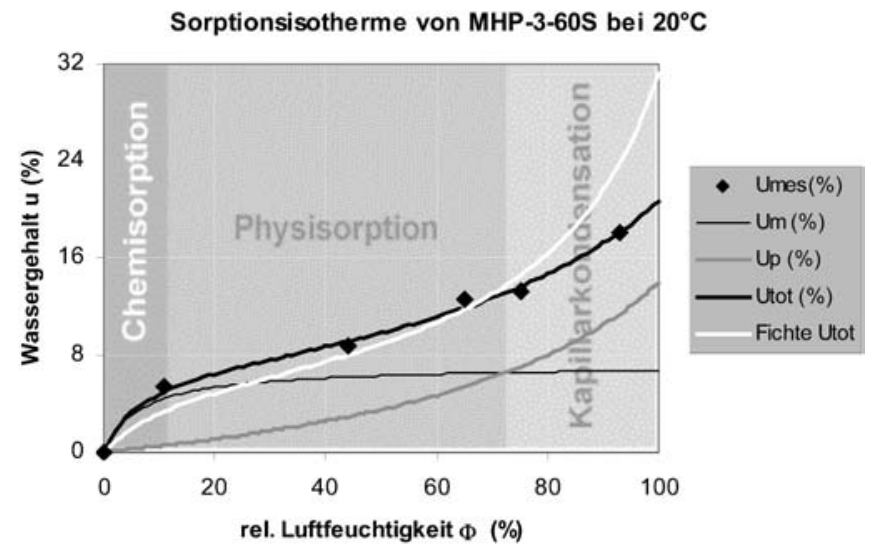

Abb. 3 Sorptionsisotherme von 3-schichtigen (Lamellenverhältnis 20/20/20), $60 \mathrm{~mm}$ dicken Massivholzplatten in senkrechter Anordnung bei $20^{\circ} \mathrm{C}$

Fig. 3 Sorption isotherm of three layer solid wood panels (layer arrangement 20/20/20), $60 \mathrm{~mm}$ thick perpendicularly arranged at $20^{\circ} \mathrm{C}$

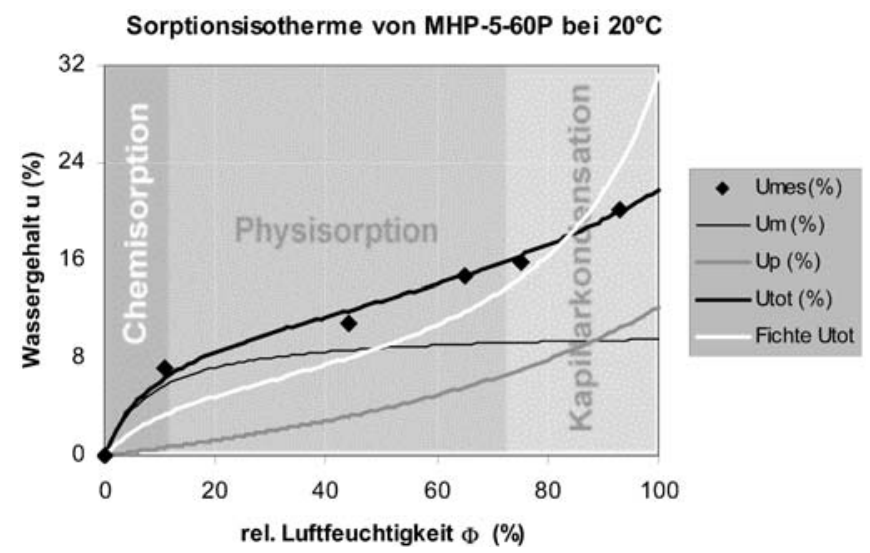

Abb. 4 Sorptionsisotherme von 5-schichtigen (Lamellenverhältnis 12/12/12/12/12), $60 \mathrm{~mm}$ dicken Massivholzplatten in paralleler Anordnung bei $20^{\circ} \mathrm{C}$

Fig. 4 Sorption isotherm of five-layer solid wood panels (layer arrangement 12/12/12/12/12), $60 \mathrm{~mm}$ thick parallelly arranged at $20^{\circ} \mathrm{C}$

Erhöhung der Sorbat-Zugänglichkeit von Platten mit parallel angeordneten Lamellen in den Decklagen gegenüber denjenigen in der senkrechter Anordnung ist mit Ausnahme von MHP-3-60A Proben im Bereich der

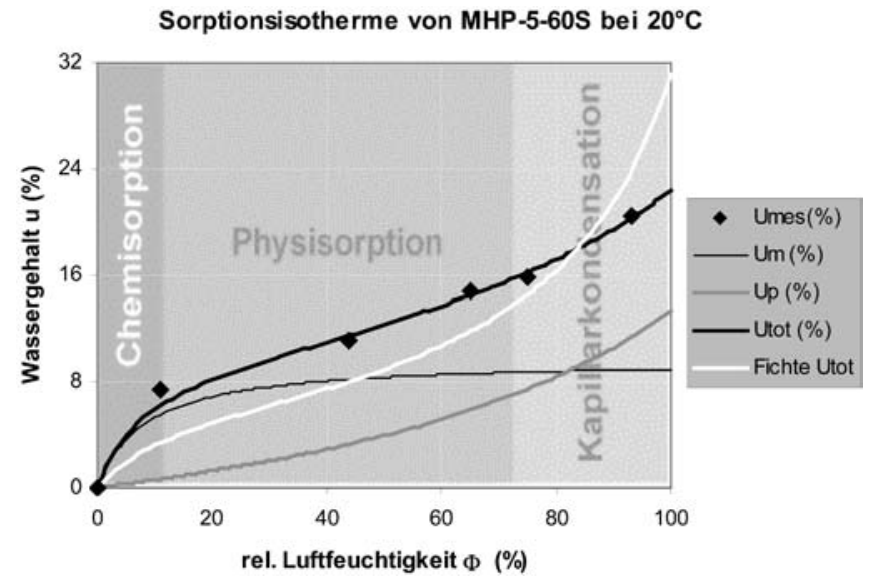

Abb. 5 Sorptionsisotherme von 5-schichtigen (Lamellenverhältnis 12/12/12/12/12), $60 \mathrm{~mm}$ dicken Massivholzplatten in senkrechter Anordnung bei $20^{\circ} \mathrm{C}$

Fig. 5 Sorption isotherm of five-layer solid wood panels (layer arrangement 12/12/12/12/12), $60 \mathrm{~mm}$ thick perpendicularly arranged at $20^{\circ} \mathrm{C}$

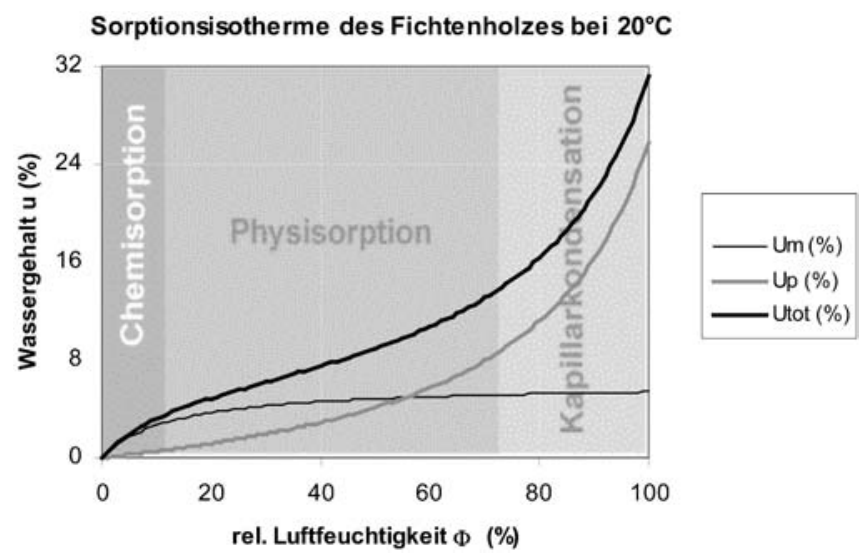

Abb. 6 Sorptionisotherme von Fichtenholz

Fig. 6 Sorption isotherm of spruce wood

monomolekularen Bedeckung $\left(U_{m}\right)$ erkennbar. Auffallend ist die relativ hohe monomolekulare Bedeckung und die relativ tiefe Fasersättigungsfeuchte aller Versuchsproben in Vergleich zu unbehandeltem Fichtenholz (siehe Abb. 6). Dies deutet darauf hin, das es durch den Herstellungsprozess einerseits zur Erhöhung der Zugäng-
Tabelle 3 Gleichgewichtsfeuchtigkeit $u$ des Versuchsmaterials entlang der Sorptionsisotherme

Table 3 Equilibrium moisture content $u$ of the material along the sorption isotherm

\begin{tabular}{llcccc}
\hline Variante & \multicolumn{5}{l}{ Holzfeuchte $u$ in $\%$ bei rel .Luftfeuchte $\Phi$ in $\%$} \\
\cline { 2 - 5 } & $\Phi=11 \%$ & $\Phi=44 \%$ & $\Phi=65 \%$ & $\Phi=75 \%$ & $\Phi=93 \%$ \\
\hline MHP-3-60P & 7,19 & 10,86 & 14,75 & 15,87 & 20,12 \\
MHP-3-60S & 5,50 & 8,78 & 12,79 & 13,31 & 18,24 \\
MHP-3-27P & 7,86 & 11,31 & 15,27 & 15,97 & 20,18 \\
MHP-3-27S & 7,48 & 11,05 & 14,85 & 15,88 & 20,41 \\
MHP-3-30P & 6,48 & 9,67 & 13,06 & 13,67 & 18,58 \\
MHP-3-30S & 5,48 & 9,18 & 13,00 & 13,62 & 18,53 \\
MHP-3-60AP & 6,45 & 9,57 & 13,45 & 14,11 & 18,72 \\
MHP-3-60AS & 5,30 & 8,88 & 12,70 & 13,89 & 19,22 \\
MHP-5-60P & 5,93 & 9,13 & 12,77 & 13,40 & 18,59 \\
MHP-5-60S & 5,11 & 8,69 & 12,61 & 13,59 & 15,56 \\
Fichtenholz & 3,40 & 8,04 & 11,79 & 14,54 & 23,79 \\
\hline
\end{tabular}


Tabelle 4 Berechnete Werte der Sorptionsanalyse nach dem Hailwood-Horrobin Modell bei Fasersättigung

Table 4 Calculated values of the sorption of the application of Hailwood Horrobin theory on saturation point

\begin{tabular}{lllllll}
\hline Probe & $\Sigma \mathrm{m}^{2} / \mathrm{g}$ & $Z \%$ & $M p$ & $U_{F S}$ & $U_{P}$ & $U_{M}$ \\
\hline MHP-3-27P & 265 & 28,23 & 226 & 20,49 & 13,01 & 7,47 \\
MHP-3-27S & 249 & 32,02 & 238 & 21,06 & 14,03 & 7,04 \\
MHP-3-30P & 269 & 26,82 & 221 & 20,86 & 13,27 & 7,60 \\
MHP-3-30S & 235 & 36,16 & 254 & 22,43 & 15,79 & 6,64 \\
MHP-3-60P & 315 & 13,34 & 187 & 22,18 & 13,30 & 8,88 \\
MHP-3-60S & 239 & 35,29 & 250 & 20,69 & 13,96 & 6,73 \\
MHP-3-60AP & 248 & 32,98 & 242 & 20,82 & 13,83 & 6,99 \\
MHP-3-60AS & 267 & 25,94 & 219 & 17,60 & 10,06 & 7,54 \\
MHP-5-60P & 339 & 6,41 & 173 & 21,77 & 12,21 & 9,56 \\
MHP-5-60S & 318 & 12,78 & 186 & 22,34 & 13,38 & 8,96 \\
Fichtenholz & 190 & 45,55 & 298 & 31,16 & 25,79 & 5,37 \\
\hline
\end{tabular}

lichkeit des Sorbats zu den sorptiv aktiven Gruppen des Holzes gekommen ist, andererseits wird die innere Struktur des Holzes teilweise durch den Leim verstopft und zusätzlich die Porenstruktur durch die prozessbedingte Druck- und Temperaturanwendung verändert. Auch ein Einfluss der sich bei Feuchteänderung zwischen den Lagen ausbildenden inneren Spannungen (bedingt durch die kreuzweise Verleimung) auf das Sorptionsverhalten dürfte vorhanden sein. Insgesamt ist die Fasersättigungsfeuchte der Platten im Vergleich zum Fichtenholz trotz der höheren Affinität der sorptiv aktiven Gruppen im Bereich der Chemisorption deutlich herabgesetzt. Die Gleichgewichtsfeuchtigkeit der Massivholzplatten ist im unteren hygroskopischen Bereich (bis etwa 65\%) bedeutend höher und im obersten Bereich bis 93\%) bedeutend tiefer als beim Fichtenholz.

\subsection{Feuchteverformung}

\subsubsection{Lineare Quellung entlang der Sorptionsisotherme}

Die Messungen haben gezeigt, dass die Längen- sowie die Breitenquellung gegenüber der Dickenquellung der Massivholzplatten klein sind, sodass die Volumenquellung der Dickenquellung nahezu gleichgestellt werden kann (Abb. 7, 8, 9, 10, 11, 12, 13, 14, 15, 16, 17, 18, 19, 20 und 21). Die Volumenquellung der Massivholzplatten liegt unter der von Vollholz. Zwischen der Holzfeuchte und der Volumenquellung besteht eine lineare Abhängigkeit (siehe z. B. Abb. 9) im Bereich zwischen $0 \%$ und etwa $20 \%$.

Aus dem Verlauf der Dickenquellung der Massivholzplatten und der Volumenquellung des Fichtenholzes im gesamten hygroskopischen Bereich lassen sich folgende Schlüsse ziehen:

1. Im unteren Dampfdruckbereich quellen die Massivholzplatten stärker als Fichtenholz.

2. Im oberen Dampfdruckbereich zeigen die Massivholzplatten ein geringeres Quellverhalten als Fichtenholz.

3. Parallel zum Sorptionsverhalten zeigen sich die Massivholzplatten dort, wo das Wasser an das Material chemisch gebunden ist, als formunstabiler (grössere Quellung), hingegen im Dampfdruckbereich, wo die
Feuchteverformung von Massivholzplatten

MHP-3-60P, Lamellenverhältnis 20/20/20 mm parallel

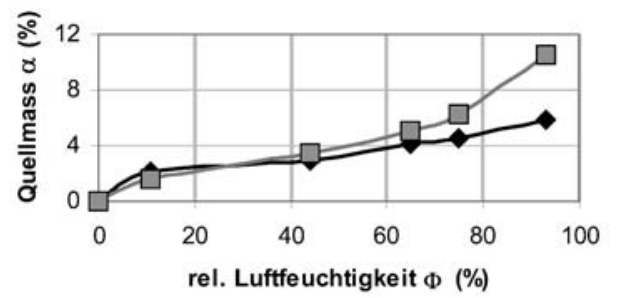

$\rightarrow$ Dicke MHP Fichtenholz

Abb. 7 Feuchteverformung von MHP-3-60P entlang der Sorptionsisotherme

Fig. 7 Swelling of the MHP-3-60P along the sorption isotherm

Feuchteverformung von Massivholzplatten

MHP-3-60S, Lamellenverhältnis 20/20/20 mm senkrecht
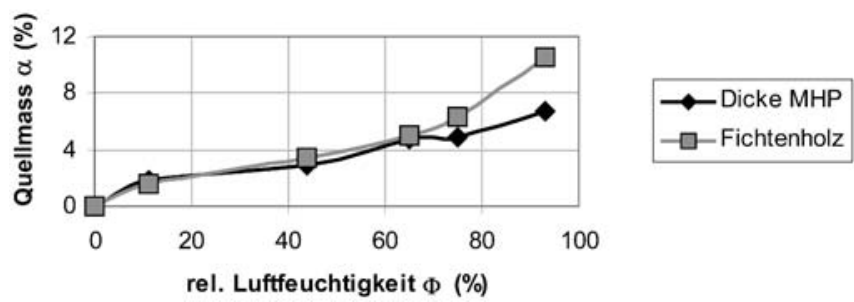

Abb. 8 Feuchteverformung von MHP-3-60S entlang der Sorptionsisotherme

Fig. 8 Swelling of the MHP-3-60S along the sorption isotherm

\section{Feuchtevervormung von Massivholzplatten} MHP-3-60, Lamellenverhältnis 20/20/20 mm

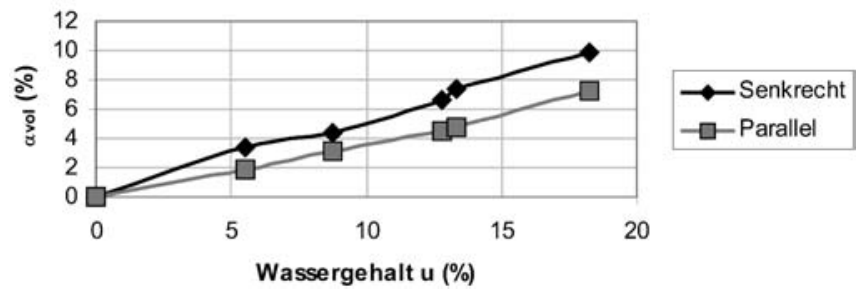

Abb. 9 Feuchteverformung von MHP-3-60 in Abhängigkeit vom Wassergehalt

Fig. 9 Swelling of the MHP-3-60 as a function of moisture content

Porenstruktur für die Wasseraufnahme maßgebend ist, als dimensionsstabiler gegenüber dem Fichtenholz (geringere Quellung).

Die Längen- und Breitenfeuchteverformungen der Proben weisen je nach Platten- und Lamellenanordnungsart an den Stirnflächen plastische Verformungen (Abb. 23) auf, die bei den relativ kleinen Probenabmessungen zu ungewöhnlich hohen Feuchteverformungen geführt haben. An den Rändern kommt es im Gegensatz zum Platteninneren zu einer nahezu freien Quellung (Herausquellung der liegenden Lagen durch die wesentlich höheren Quellmaße). Diese plastischen Verformungen werden sich bei den großformatigen Platten auf die Längen- und Breitenfeuchteverformung kaum auswirken, es treten dadurch 
Feuchteverformung von Massivholzplatten

MHP-5-60P, Lamellenverhältnis 12/12/12/12/12 mm paralle

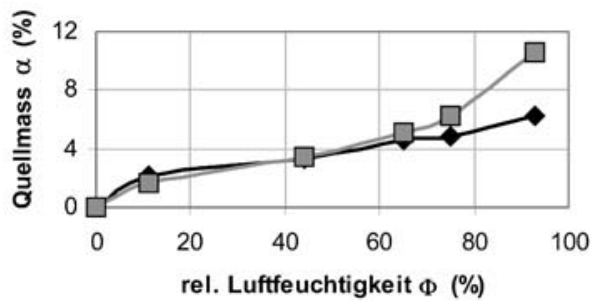

Abb. 10 Feuchteverformung von MHP-5-60P entlang der Sorptionsisotherme

Fig. 10 Swelling of the MHP-5-60P along the sorption isotherm

Feuchteverformung von Massivholzplatten

MHP-5-60S, Lamellenverhältnis 12/12/12/12/12 mm senkrecht
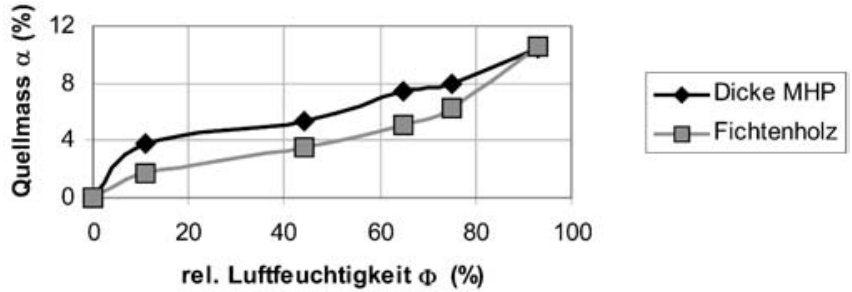

Abb. 11 Feuchteverformung von MHP-5-60S entlang der Sorptionsisotherme

Fig. 11 Swelling of the MHP-5-60S along the sorption isotherm

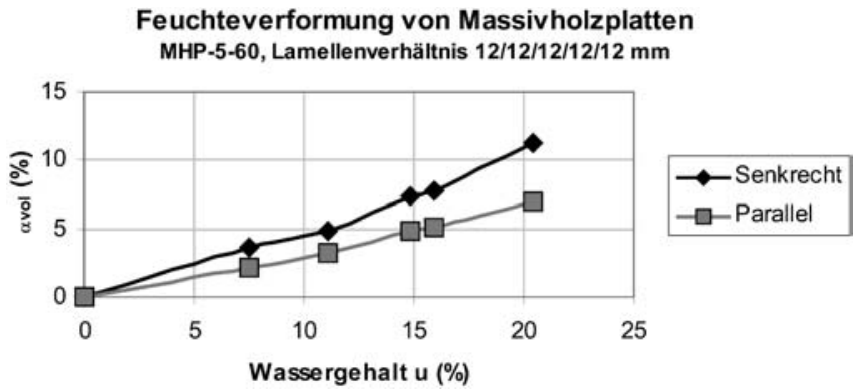

Abb. 12 Feuchteverformung von MHP-5-60 in Abhängigkeit vom Wassergehalt

Fig. 12 Swelling of the MHP-5-60 as a function of moisture content

aber erhebliche Spannungen zwischen den Schichten auf, die im Extremfall zu Verformungen der Platten (Plattenverzug) oder auch zu Rissen führen können.

\subsubsection{Dickenquellung als Funktion der Anzahl von Leimschichten pro $\mathrm{mm}$ Plattendicke}

Abbildung 22 zeigt die Abhängigkeit der Dickenquellung der Versuchsproben von der Anzahl Leimschichten pro mm Plattendicke. Wie aus diesen Abbildungen hervorgeht, steigt die Quellung der Massivholzplatten linear mit
Feuchteverformung von Massivholzplatten

MHP-3-27P, Lamellenverhältnis 6/15/6 mm parallel, beschichtet
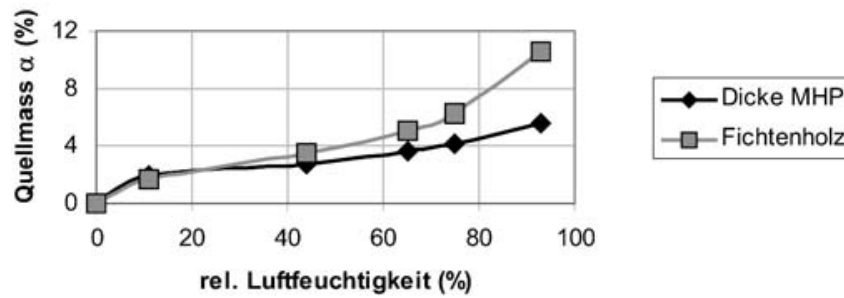

Abb. 13 Feuchteverformung von MHP-3-27P entlang der Sorptionsisotherme

Fig. 13 Swelling of the MHP-3-27P along the sorption isotherm

Feuchteverformung von Massivholzplatten

MHP-3-27S, Lamellenverhältnis $6 / 15 / 6 \mathrm{~mm}$ senkrecht, beschichtet
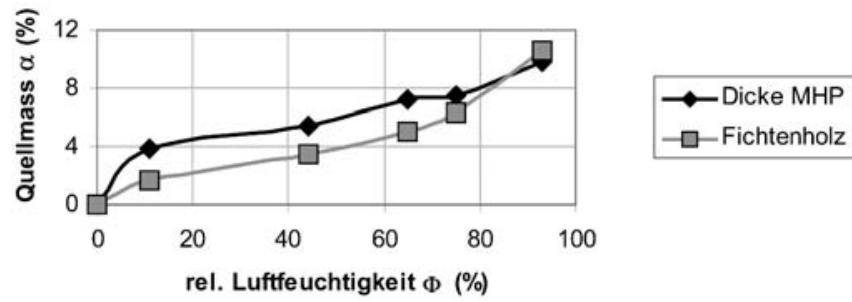

Abb. 14 Feuchteverformung von MHP-3-27S entlang der Sorptionsisotherme

Fig. 14 Swelling of the MHP-3-27S along the sorption isotherm

Feuchteverformung von Massivholzpatten MHP-3-27, Lamellenverhältnis 6/15/6 mm senkrecht, beschichtett

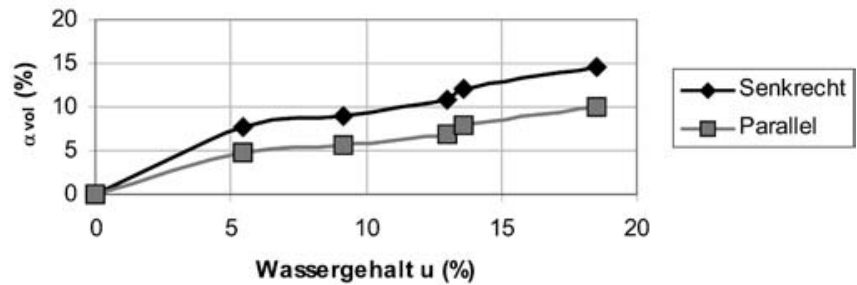

Abb. 15 Feuchteverformung von MHP-3-27 in Abhängigkeit vom Wassergehalt

Fig. 15 Swelling of the MHP-3-27 as a function of moisture content

der Anzahl Leimschichten pro mm Plattendicke. Diese Abhängigkeit ist für den untersuchten hygroskopischen Bereich zwischen rel. Luftfeuchtigkeit von $11 \%$ bis $93 \%$ statistisch gesichert.

Aus dieser Abbildung ist weiter ersichtlich, dass die Steigung der Geraden vom Bereich der Chemi- über Physisorption bis zum Bereich der Kapillarkondensation stetig ansteigt. 
Feuchteverformung von Massivholzplatten MHP-3-30P, Lamellenverhältnis $10 / 10 / 10 \mathrm{~mm}$ parallel
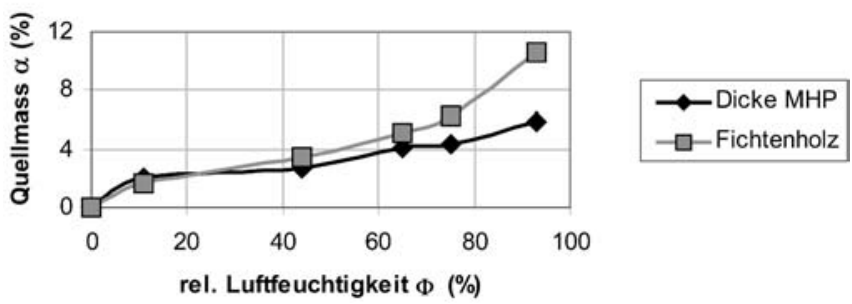

Abb. 16 Feuchteverformung von MHP-3-30P entlang der Sorptionsisotherme

Fig. 16 Swelling of the MHP-3-30P along the sorption isotherm

Feuchteverformung von Massivholzplatten

MHP-3-30S, Lamellenverhältnis 10/10/10 mm senkrecht
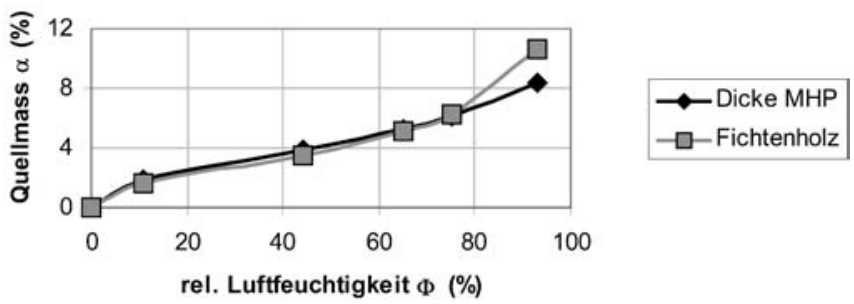

Abb. 17 Feuchteverformung von MHP-3-30S entlang der Sorptionsisotherme

Fig. 17 Swelling of the MHP-3-30S along the sorption isotherm

Feuchteverformung von Massivholzplatten MHP-3-30, Lamellenverhältnis 10/10/10 mm
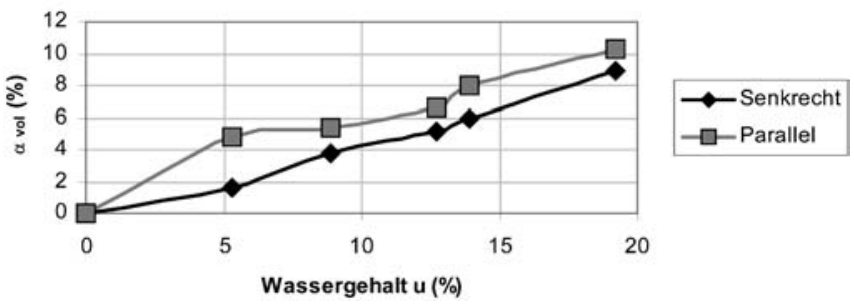

Abb. 18 Feuchteverformung von MHP-3-30 in Abhängigkeit vom Wassergehalt

Fig. 18 Swelling of the MHP-3-30 as a function of moisture content

\subsubsection{Differentielle Dickenquellung}

Tabelle 5 zeigt die differentielle Dickenquellung. Diese wurde für drei Feuchteänderungen von rel. Luftfeuchtigkeiten zwischen $93 \%$ und $44 \%, 75 \%$ und $44 \%, 75 \%$ und $11 \%$ ermittelt. Die Werte liegen im Bereich von 0,3$0,5 \% / \%$ Feuchteänderung.

Die differentielle Dickenquellung der Massivholzplatten bei senkrechter Anordnung der Decklagen ist mit Ausnahme der MHP-3-60AS auffallend höher im Vergleich mit derjenigen mit paralleler Anordnung.
Feuchteverformung von Massivholzplatten

MHP-3-60AP, Lamellenverhältnis 10/40/10 mm parallel

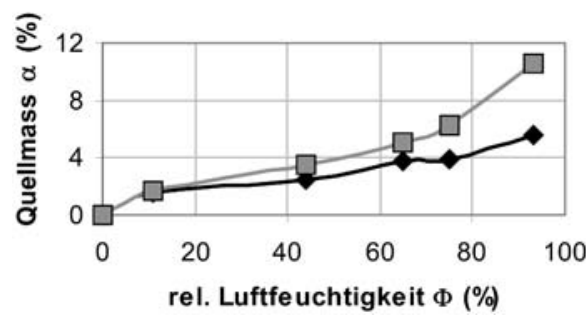

$\longrightarrow$ Dicke MHP

Fichtenholz

Abb. 19 Feuchteverformung von MHP-3-60AP entlang der Sorptionsisotherme

Fig. 19 Swelling of the MHP-3-60AP along the sorption isotherm

Feuchteverformung von Massivholzplatten MHP-3-60AS, Lamellenverhältnis 10/40/10 mm senkrecht

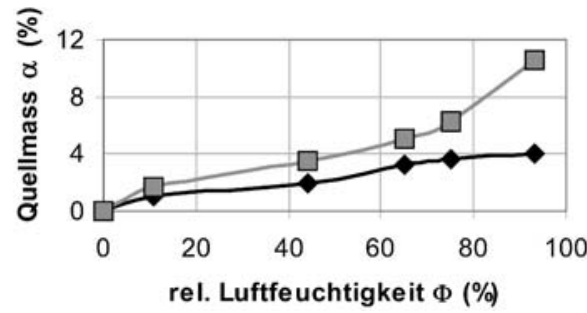

$\longrightarrow$ Dicke MHP

$\square-F i c h t e n h o l z$

Abb. 20 Feuchteverformung von MHP-3-60AS entlang der Sorptionsisotherme

Fig. 20 Swelling of the MHP-3-60AS along the sorption isotherm

Feuchteverformung von Massivholzplatten

MHP-3-60A, Lamellenverhältnis 10/40/10 mm

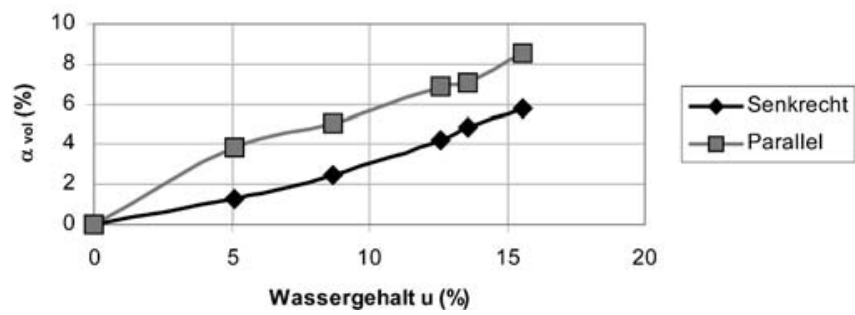

Abb. 21 Feuchteverformung von MHP-3-30 in Abhängigkeit vom Wassergehalt

Fig. 21 Swelling of the MHP-3-30 as a function of moisture content

\subsubsection{Differentielle Längenquellung}

Bei der $60 \mathrm{~mm}$ dicken dreilagigen Platte MHP-3-60 beträgt die Dickenquellung $0,045 \% / \%$, bei der $60 \mathrm{~mm}$ dicken, fünflagigen $0,021 \% / \%$, bei der $60 \mathrm{~mm}$ dicken dreilagigen (MHP60 A (10/40/10)) 0,016\%/\% und bei der $27 \mathrm{~mm}$ dicken Betonschaltafel 0,015\%/\%. Dabei erfolgte die Längenmessung jeweils an den längsliegenden Lagen (in Faserrichtung). Es zeigt sich, dass bei hoher Feuchte erhebliche Spannungen in den Lagen auftreten und dass es an den Rändern zu plastischen Verformungen (Heraus- 
Tabelle 5 Differentielle Dickenquellung $q$ in $\% / \%$ bei drei verschiedenen rel. Luftfeuchteänderungen in $\%$ Table 5 Differential thickness swelling $q(\% / \%)$ at various changes of relative humidity in $\%$

\begin{tabular}{lllll}
\hline Probe & $\Delta \Phi=44 \%-93 \%$ & $\Delta \Phi=44 \%-75 \%$ & $\Delta \Phi=11 \%-75 \%$ & Rohdichte $\rho\left(\mathrm{kg} / \mathrm{m}^{3}\right)$ \\
\hline MHP-3-27P & 0,32 & 0,35 & 0,31 & 450 \\
MHP-3-27S & 0,47 & 0,47 & 0,45 & 450 \\
MHP-3-30P & 0,35 & 0,35 & 0,30 & 450 \\
MHP-3-30S & 0,43 & 0,45 & 0,50 & 460 \\
MHP-3-60P & 0,32 & 0,31 & 0,27 & 440 \\
MHP-3-60S & 0,40 & 0,46 & 0,39 & 480 \\
MHP-3-60AP & 0,32 & 0,33 & 0,31 & 420 \\
MHP-3-60AS & 0,29 & 0,33 & 0,30 & 420 \\
MHP-5-60P & 0,34 & 0,34 & 0,33 & 440 \\
MHP-5-60S & 0,54 & 0,55 & 0,50 & 480 \\
\hline
\end{tabular}

Dickenquellung von Massivholzplatten entlang der Sorptionsisotherme

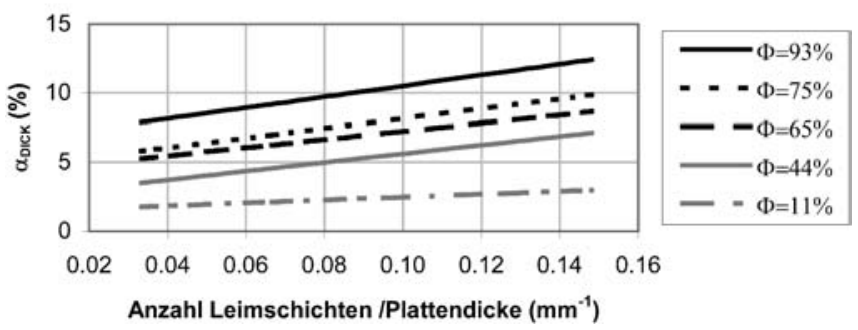

Abb. 22 Einfluss der Anzahl der Leimfugen je mm Plattendicke auf das Volumenquellmaß

Fig. 22 The influence of the number of glued joint per $\mathrm{mm}$ of the panel thickness on the volume swelling

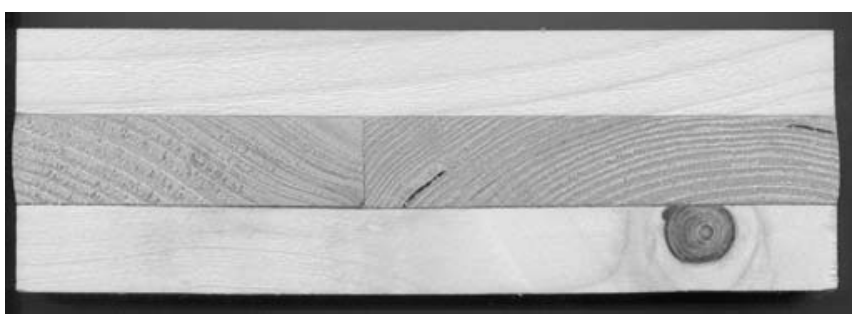

Abb. 23 Herausquellen der querliegenden Lagen im Feuchtklima bei $93 \%$ rel. Luftfeuchte

Fig. 23 Swelling out of the transverse situated lamellas in the wet climate at $93 \%$ relative humidity

quellen der senkrecht liegenden Lagen mit deutlich höherem Quell- und Schwindmaß) kommt (Abb. 23). Ferner ist ein Einfluss des Plattenaufbaus (Verhältnis der Lagendicken) und offensichtlich auch des Schlitzens der Mittellagen vorhanden, der näher untersucht werden muss. Die Kennwerte der differentiellen Quellung liegen im Bereich der aus Firmenschriften bekannten Werte. Eine gewisse Variabiltät ergibt sich durch den unterschiedlichen Plattenaufbau. Der Einfluss des Plattenaufbaus sollte in weiterführenden Arbeiten untersucht werden.

\section{Schlussfolgerungen}

Aufgrund der erzielten Resultate lassen sich folgende Schlüsse ziehen:
Gleichgewichtsfeuchtigkeit

- Die Gleichgewichtsfeuchtigkeit der Massivholzplatten ist im unteren hygroskopischen Bereich (bis etwa $65 \%$ ) bedeutend höher und im obersten Bereich (93\%) bedeutend tiefer als diejenige beim Fichtenholz.

\section{Sorptionseigenschaften}

- Es besteht kein statistisch relevanter Unterschied zwischen den Sorptionseigenschaften der untersuchten Massivholzplatten.

- Eine tendentielle Erhöhung der Sorbat-Zugänglichkeit von Platten mit parallel angeordneten Lamellen gegenüber denjenigen mit der senkrechter Anordnung ist mit Ausnahme von MHP-3-60A Proben zu verzeichnen.

- Auffallend ist die relativ hohe monomolekulare Bedeckung und die relativ tiefe Fasersättigungsfeuchte aller Versuchsproben im Vergleich zu unbehandeltem Fichtenholz. Dies deutet darauf hin, dass es durch den Herstellungsprozess einerseits zur Erhöhung der $\mathrm{Zu}$ gänglichkeit des Sorbats zu den sorptiv aktiven Gruppen des Holzes gekommen ist, andererseits wurde die innere Struktur des Holzes teilweise durch den Klebstoff verstopft und möglicherweise die Porenstruktur durch prozessbedingte Druck- und Temperaturanwendung verändert.

- Im Endeffekt ist die Fasersätigungsfeuchte der Platten im Vergleich zum Fichtenholz deutlich herabgesetzt. Demnach setzt sich der Sorptionsmechanismus der Kapillarkondensation gegenüber der Chemisorption auf die Fasersättigungsfeuchte stärker durch.

\section{Lineare Quellung}

- Die Messungen haben gezeigt, dass die Längen- sowie die Breitenquellung gegenüber der Dickenquellung der Massivholzplatten erwartungsgemäss vernachlässigbar klein ausfallen, sodass die Volumenquellung der Dickenquellung gleichgestellt werden kann.

- Das Quellen im Bereich zwischen 0\% und etwa 20\% Wassergehalt ist nahezu linear und die Beziehung zwischen Quellen und rel. Luftfeuchtigkeit kann der Sorptionsisotherme annähernd gleichgestellt werden. 
- Aus dem Verlauf der Dickenquellung der Massivholzplatten und der Volumenquellung des Fichtenholzes im gesamten hygroskopischen Bereich lassen sich folgende Schlüsse ziehen:

- Im unteren Dampfdruckbereich quellen die Massivholzplatten ausnahmslos stärker als das Fichtenholz.

- Im oberen Dampfdruckbereich zeigen die Massivholzplatten ein geringeres Quellverhalten gegenüber dem Fichtenholz.

- Parallel zum Sorptionsverhalten zeigen sich die Massivholzplatten dort, wo das Wasser an das Material chemisch gebunden ist formunstabiler, hingegen im Dampfdruckbereich, wo die Porenstruktur für die Wasseraufnahme maßgebend ist dimensionsstabiler gegenüber dem Fichtenholz.

- Die Längen- und Breitenfeuchteverformungen der Versuchsproben weisen je nach Platten- und Lamellenanordnungsart an den Stirnflächen plastische Verformungen auf, die bei den relativ kleinen Probenabmessungen $\mathrm{zu}$ ungewöhnlich hohen Feuchteverformungen geführt haben. Hier wäre eine Messung an der Oberfläche (Verschiebung der Messmarken) sinnvoller, als eine Messung an den Probenenden. Diese plastische Verformungen werden sich bei den grossen Industrieplatten auf die Längen- und Breitenfeuchteverformung kaum auswirken.

- Die Quellung der Massivholzplatten steigt linear mit der Anzahl der Leimschichten pro Plattendicke.

- Je höher das Feuchteklima, desto höher ist auch die Dickenquellung.

- Die Steigung der Geraden (Dickenquellung in Abhängigkeit von der Anzahl Leimschichten pro Plattendicke) steigt vom Bereich der Chemi- über die Physisorption bis zum Bereich der Kapillarkondensation stetig an.

\section{Differentielle Quellung}

- Die differentielle Dickenquellung der Massivholzplatten mit senkrechter Lamellenanordnung ist mit Ausnahme der MHP-3-60AS auffallend höher im Vergleich mit derjenigen mit der parallelen Anordnung. Sie liegt im Bereich von 0,3 bis $0,5 \% / \%$. Die differentielle Längenquellung liegt zwischen 0,016 bis $0,045 \% / \%$.

\section{Literatur}

DIN 52182 (1976) Prüfung von Holz. Bestimmung der Rohdichte. S. 1-2

DIN 52183 (1977) Prüfung von Holz. Bestimmung des Feuchtigkeitsgehaltes. S. 1-2

DIN 52184 (1979) Prüfung von Holz. Bestimmung der Quellung und Schwindung. S. 1-4

DIN 50014 (1985) Klimate und ihre technische Anwendung. Normalklimate. S. 1-2

Hailwood A, Horrobin S (1946) Absorption of water by polymers. Analysis in term of a single model. Trans Faraday Soc 42B:84102

Krug D, Tobisch S, Faust E (1999a) Massivholzplatten für konstruktive Anwendungen. Teil 1. Holz-Zentralblatt 125(94): $1282-1283$

Krug D, Tobisch S, Faust E (1999b) Massivholzplatten für konstruktive Anwendungen. Teil 2. Holz-Zentralblatt 125(100): $1335-1336$

Lignum (1997) Zürich, Holzprodukte für den statischen Einsatz. Teil 1. Plattenförmige Werkstoffe. Herstellung von 3-lagigen Massivholzplatten unter gezielter Variation der Lamellenfeuchte. Interner Bericht IHD, Dresden. Tagungsband. 3. IHD-Holzwerkstoff-Kolloqium. Institut für Holztechnologie, Dresden, 9 Januar 1999

Niemz P, Poblete H (1995)Untersuchungen zur Gleichgewichtsfeuchte von MDF und Spanplatten. Holz Roh- Werkstoff 53(6):368

Niemz P, Poblete H (1996) Untersuchungen zur Dimensionsstabilität von mitteldichten Faserplatten (MDF) und Spanplatten. Holz Roh- Werkstoff 54(2):141-144

Niemz P, Popper R, Eberle G (2002) Untersuchungen zu mechanisch-physikalischen Eigenschaften mehrschichtiger Massivholzplatten für das Bauwesen. Holz 1:33-36

Noack D, Schwab E (1986) In: von Halasz R, Scheer R (Hrsg) Holzbautaschenbuch. Ernst Verlag für Architektur und Technischen Wissenschaften, Berlin

Pius Schuler AG, Blockholz. Firmenmaterial, Rothenthurm

Popper R (1982) Computerprogramm HHM28 für die Berechnung von Sorptionseigenschaften nach dem Hailwood-Horrobin Modell. Computer-Anwendungen: 113

Radovic B, Cheret P, Heim F (1997) Holzbau Handbuch. Informationsdienst, Holz, Düsseldorf

Suchsland O, Xu H (1991) The expansion potential:a new evaluator of expansion behavior of wood composietes. Forest Prod J 41:39-42

Sell J (1989) Eigenschaften und Kenngrössen von Holzarten. Lignum, Zürich 